\title{
Research on Sunshine Temperature Distribution in Reinforced Concert Silo by finite element method
}

\author{
Liang Zhao ${ }^{1, \text { a }}$, Zhiyong Yang ${ }^{2, b}$ \\ ${ }^{1}$ Department of Civil and Architecture Engineering, Wuhan University of Technology Huaxia College, \\ Wuhan, China \\ ${ }^{2}$ School of Civil Engineering and Architecture, Wuhan University of Technology, Wuhan, China \\ azhaolianghj16@163.com, byzy0807@126.com
}

Keywords: reinforced concert silo, temperature distribution, sunshine, finite element analysis

\begin{abstract}
Sunshine one of the most severe factors makes great effects on the mass concrete construction, especially on larger reinforced concert silo. In order to calculate the temperature distribution of the reinforced concrete silo subjected to the solar radiation, an easy-to-use tool is required for a transient analysis. As the first step of this paper, large-scale finite element software ANSYS is chosen as a basic platform. Further, the ANSYS Parameter Design Language (APDL) is adopted to establish a program to predict the nonlinear temperature distributions in circular reinforced concert silo in case of the solar radiation based on the experimental data and the heat transfer theory. Finally, the effect of the solar radiation, the geographic location as well as geometrical dimensions is investigated. The results clarity the distribution characters of the temperature. Besides, computational and experimental results are compared. The results indicate that sunshine nonlinearity temperature different can reach up to $10.2^{\circ} \mathrm{C}$. As a result, attention should be paid to such temperature effect on the circular reinforced concrete silo.
\end{abstract}

\section{Introduction}

Silo is a storage structure, which is generally used for the storage of loose graininess, patches raw materials or fuel and so on. With the development of industrial, the diameter and height of silo has been increased conspicuously. There are a mass of accident about damaged silo ${ }^{[1]}$, whose reasons are neglect of the influence of temperature stress. Larger the temperature difference is, the greater temperature stress will be produced. Temperature stress will result in great tension and even cracking in silo. So the study of temperature effect is necessary for the actual construction.

With the development of numerical methods, especially the finite-element method, many papers relevant to temperature have been published in recent years. Jiang, Shufen et al. (1987) ${ }^{[2]}$ used finite element software to analyze temperature distribution under the solar radiation on the condition of full silo, which was compared with measured results. J.Y.Ooi et al. (1990) ${ }^{[3]}$ made use of Lade model to study the effects of the static pressure of the barn and the influence of temperature. Pu Weiming et al. (1999) ${ }^{[4]}$ researched the effects of seasonal temperature difference, internal and external temperature difference and sunshine temperature difference on reinforced concrete silo. Xia Guangzheng, Xia Dongmei (2006) ${ }^{[5]}$ analyzed a diameter of 120 giant silo subject to environmental temperature, and proposed the normal function of environmental temperature to concrete silo wall, which is relevant to diameter silo instead of depth of silo. Fang Hongqiang et al. (2011) ${ }^{[6]}$ proposed the temperature field method about basing on thermodynamic principle to solve the concrete structure. YangYinghua, ZangYan et al. (2012) ${ }^{[7]}$ analyzed and studied two aspects of strength and stability of concrete silo under the influence of sunshine temperature in different seasons. CuiYuanrui et al. (2012) ${ }^{[8]}$ researched the principle and calculation method about temperature effect of reinforced concrete silo, and pointed out that the large diameter or super diameter must be checked the effect of sunshine temperature difference.

Most studies of concrete silo with temperature are for specific projects, and more of them are steady state analysis, which aren't analyzed in detail. Based on experiment, the FEM software ANSYS is taken to simulate temperature effects under sunshine in this paper. The temperature 
distribution is obtained, which could provide reference for the further analysis of temperature stress of the silo.

\section{Transient Thermal Analysis Theory}

Transient heat transfer process is a heating or cooling process. Temperature, heat flow rate, thermal boundary conditions and internal energy of the system are changed with time obviously. In numerical calculation of the transient thermal problem, the node temperature varies with the position and time. According to the view of the energy balance, grid units have the heat import or export between neighboring units, and its internal energy changes with time.

According to the principle of energy conservation: transient thermal balance can be expressed in the general form (in a matrix type) ${ }^{[9]}$ :

$$
[C]\{\dot{T}\}+[K]\{T\}=\{Q\}
$$

where $[K]$ is conductance matrix, including thermal conductivity, convection coefficient, emissivity and shape factor. $[C]$ is heat capacity matrix, considering the increasing internal energy of the system. $\{T\}$ is the node temperature vector. $\{T\}$ is derivative of temperature to time. $\{Q\}$ is rate of heat flow vector of the node, including thermogenesis.

\section{Boundary condition under sunshine}

Solar radiation. The solar radiation of the surface of the structure on the ground mainly includes: direct solar radiation, diffuse radiation and reflection radiation. The vertical plane of the radiation expression is as follow:

In practical engineering application, the direct solar radiation received by the silo could be approximately obtained as:

$$
I_{D}=0.9^{t_{u} k_{a} m} I_{0} \cos \phi
$$

Where $t_{u}$ is atmospheric turbidity coefficient; $k_{a}$ is atmospheric relative pressure changed with altitude; $I_{0}=1367\left[1+0.33 \cos \left(360^{\circ} \mathrm{N} / 365\right)\right]$, it is solar constant; $N$ is daily ordinal number staring from New Year's Day; $m=1 / \sin \beta_{s}$, it is optical air mass; $\beta_{s}$ is solar azimuth angle; $\phi$ is solar incident angle.

Solar scatter intensity on Vertical Plane is:

$$
I_{H}=0.5\left(0.271 I_{0}-0.294 I_{D}\right) \sin \beta
$$

Ground reflective intensity is:

$$
I_{R}=0.5 r_{e}\left(I_{D} \sin \beta_{s}+I_{H}\right)
$$

Where $r_{e}$ is ground short-wave reflectivity.

The superposition of equations(2),(3) and (4) obtains that the total solar radiation heat flux of concrete silo wall below:

$$
q_{s}=A_{s}\left[\left(\cos \phi+0.5 r_{e} \sin \beta_{s}\right) I_{D}+0.5\left(1+r_{e}\right) I_{H}\right]
$$

Where $A_{s}$ is absorption of solar radiation.

Convective heat transfer. According to newton cooling theory, the convective heat flux between the surface and the air can be calculated as:

$$
q_{c}=h_{c}\left(T_{a}-T\right)
$$


Where $T_{a}$ is atmosphere temperature; $T$ is boundary temperature; $h_{c}$ is convective heat transfer coefficient. Considering unfavorable situation, the wind speed is $1 \mathrm{~m} / \mathrm{s}$.

\section{The establishment of boundary conditions and initial conditions}

Heat transfer boundary condition of outer surface of the silo. The heat exchange of outer surface of the silo contains radiation heat transfer caused by solar radiation, convection heat transfer between surface in the silo and atmosphere, and solar radiation energy absorbed by the surface in the silo. Because the convection and radiation loads can't be applied at the same time in ANSYS analysis. Three heat flux densities are converted to third boundary conditions:

$$
\left.\lambda \frac{\partial T}{\partial n}\right|_{\Gamma}=h\left(T_{a}^{*}-T\right)
$$

Where $T_{a}^{*}$ is synthetical air temperature; $T$ is boundary temperature; $h$ is synthetical film conefficienet.

Heat transfer boundary condition of inner surface of the silo. This paper mainly analyses the state of hollow silo which can be regarded as completely closed space. Heat exchange of the silo air and the outside was ignored, only convection heat and the radiation heat transfer between surfaces are considered. In order to simplify the calculation, a surface comprehensive heat transfer coefficient is used to express synthetically the convection and radiation heat transfer in the silo. And boundary condition is:

$$
\left.\lambda \frac{\partial T}{\partial n}\right|_{\Gamma}=h^{\prime}\left(T_{a}^{\prime}-T\right)
$$

Where $h^{\prime}$ is synthetical film conefficienet of the convection and radiation; $T_{a}^{\prime}$ is air temperature in the silo which value is approximate the average temperature inside silo.

The value of initial conditions. The initial condition is particular temperature distribution state for analysis. Generally, relatively moment of uniform temperature distribution can be chosen as the initial temperature state of the structure. The paper selects measured temperature of concrete surface about one hour before sunrise as boundary condition to analyze steady state, whose result could be regarded as the initial temperature field for transient analysis.

\section{Analysis of examples and parameters}

Finite element method. In actually, there are only two extreme states of silo: empty silo and full silo. The transition state of the partial coal in the silo is contained in the above states. Since the paper considers the effect of the sunshine, the temperature field in the empty silo is analyzed mainly. The temperature field of the silo under the sunshine belongs to transient analysis. However the temperature distribution of the silo is a three-dimensional problem. The state of empty can be considered as a tube structure without internal heat source, and temperature distribution is uniform along the height direction under the sunshine, which could be simplified as two-dimensional problem.

In the paper, a computer program module established by the APDL employing the software ANSYS as its running platform is developed to implement this model numerically. This analysis module is so generality that it's easy to study the influence of solar radiation, location, geometrical dimensions and other parameters on temperature distribution.

Model. Experimental model is a circular reinforced concert silo which diameter is $3 \mathrm{~m}$ and wall thickness is $30 \mathrm{~cm}$. The Experimental model is given in Figure 1, and the layout of measuring points is given in Figure 2. It measured continuous day in the summer. Without loss of generality, August 28, 2013 was chosen to calculate the temperature field with above parametric model. The geographical latitude is $30.62^{\circ} \mathrm{C}$, atmospheric turbidity factor is 3.97 . The absorptive of concrete surface to solar radiation is 0.55 . The range of the temperature variation of the day calculated from $6: 00$ to $19: 00$ is $29^{\circ} \mathrm{C}$ to $42^{\circ} \mathrm{C}$. The parameters are shown in table 1 . 


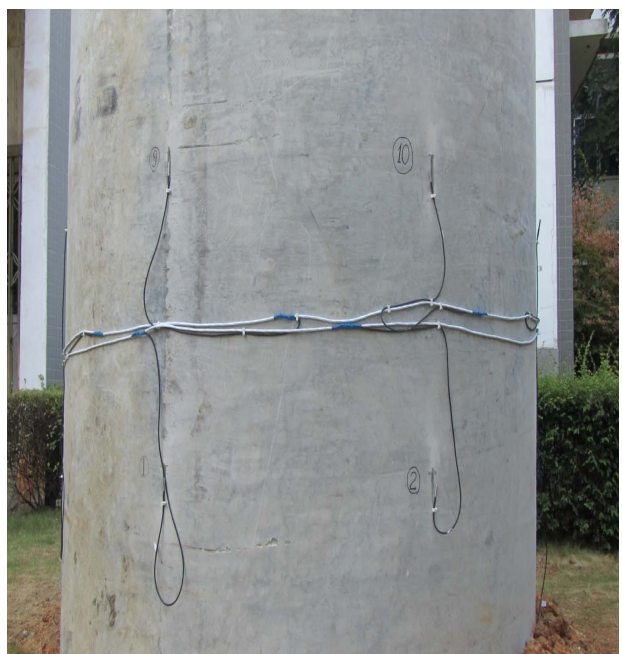

Fig. 1 Experimental model

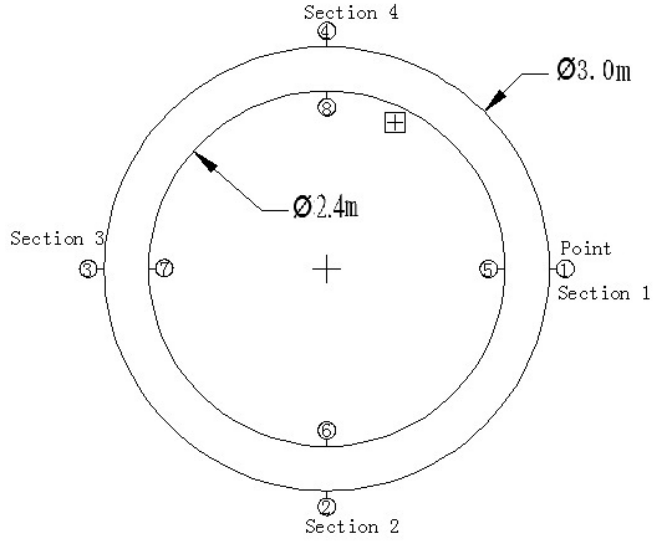

Fig.2 Distribution of measuring points

Table 1 Thermal parameters of concrete adopted in the present study

\begin{tabular}{ll}
\hline thermal conductivity $(\mathrm{W} /(\mathrm{m} \cdot \mathrm{K}))$ & 1.5 \\
\hline specific heat $(\mathrm{J} /(\mathrm{kg} \cdot \mathrm{K}))$ & 980 \\
\hline density $\left(\mathrm{kg} / \mathrm{m}^{3}\right)$ & 2400 \\
\hline
\end{tabular}

The results analysis. The temperature-time curves of different points obtained by ANSYS simulation and experiment are shown in Figure 3. The comparison shows that the temperature variation of the calculation and experiment basically tallies.

Temperature change range of each point is obvious differently. The inner points 5 to 8 increased by $8^{\circ} \mathrm{C}$, while the surface points 1 to 4 increased by $17^{\circ} \mathrm{C}$. Since the solar radiation gain by the point 2 is largest, the temperature of them increases sharply and reaches its maximum temperature $45.5^{\circ} \mathrm{Cat}$ 14:00. The solar radiation gained by the point 1 is largest at 10:00, and it reaches its maximum temperature $42.5^{\circ} \mathrm{C}$. The radiation gained by the point 4 is least, so the temperature change is small.

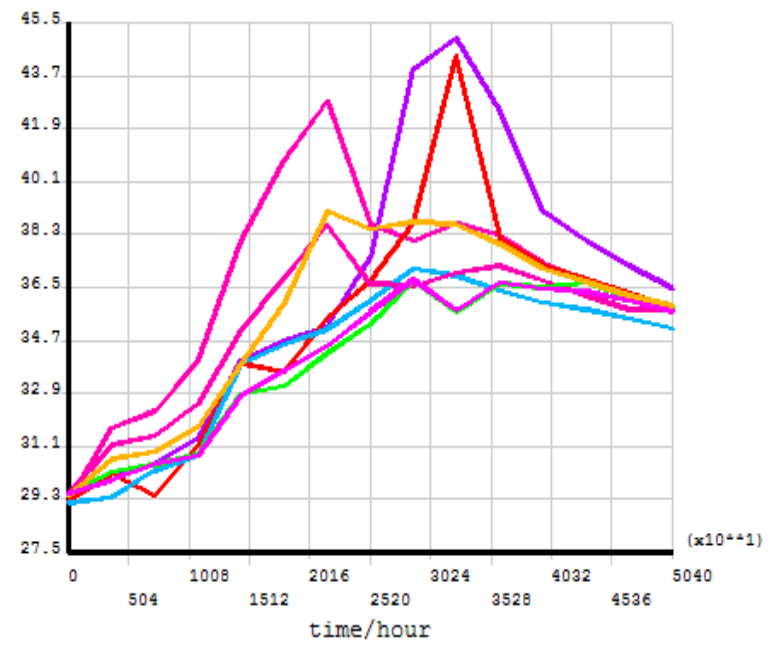

(a) Obtained by using FEM

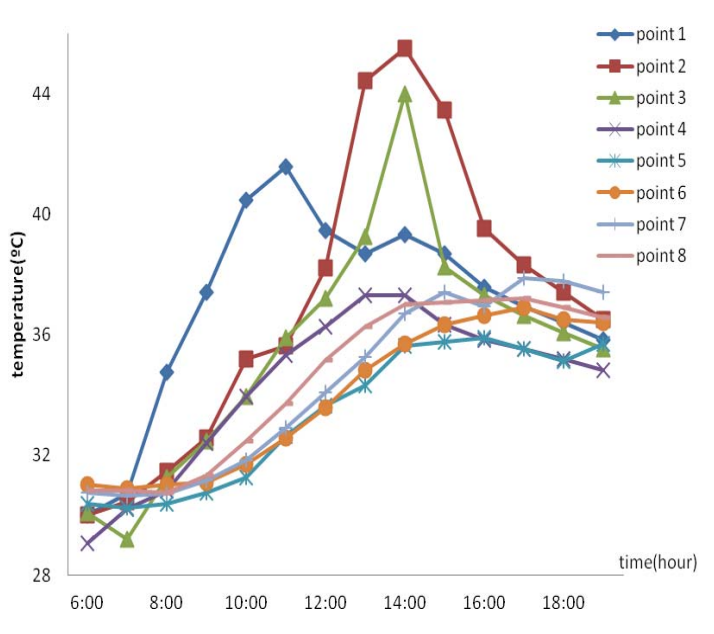

(b) Obtained by experiment

Fig.3 Temperature-time curves of each point

The temperature distribution of the silo at 14:00 is plotted in Figure 4. The nonlinear difference in temperature on the surface wall due to solar radiation can be greater $8.5^{\circ} \mathrm{C}$. 
Figure 5 shows the temperature difference variation with the time for four sections. It can be seen clearly that temperature difference of the section three is the largest; the value is $10.2^{\circ} \mathrm{C}$, which could cause larger temperature stress.
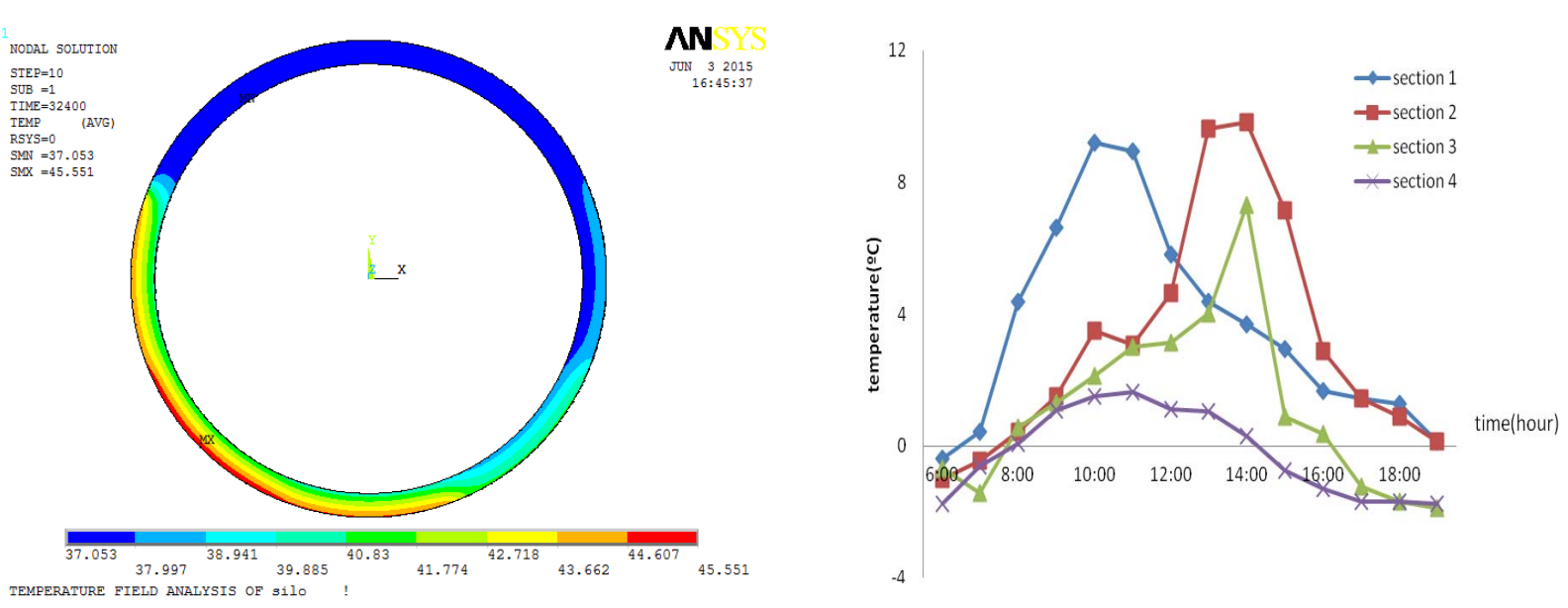

Fig.4 Temperature distribution at 14:00

Fig5. Temperature difference-time curves of each section

\section{Parameter Study}

Parametric studies are conducted to examine and discuss the effects of various parameters on the temperature distributions by the above APDL program module, and rules are obtained as following:

The influence of geographical location for the temperature distribution is more complicated. Generally, the maximum vertical difference in temperature of the cross-section appeared in summer at different latitude in our country. Under the same case, the maximum temperature difference and the surface temperature at high latitudes is larger than that of the low latitudes.

Wall thickness has great influence to the temperature distribution, when in the same conditions. With the increase of the Wall thickness, the temperature difference increases, while the maximum temperature of the surface changed little.

The influence of diameter on silo is not obvious than that of Wall thickness. The maximum temperature difference doesn't change significantly in function of the diameter. However the diameter has a significant effect on the nonlinear distribution of the cross-section in case of the same moment.

The solar radiation absorptivity of the silo surface is the most significant factor that affects the difference in temperature. When the absorptivity from 0.6 to 0.2 , the maximum temperature difference decreases from $12^{\circ} \mathrm{C}$ to $2^{\circ} \mathrm{C}$.

\section{Conclusions}

Based on the experiment, transient temperature field was analyzed by finite element method. The influences of solar radiation absorption, location and geometrical dimensions on temperature distribution are also analyzed. Compared computational results with experimental data, the summaries of conclusions are as follows:

The nonlinear temperature distribution of the cross-section is significant under sunshine. Due to the different solar radiation, maximum temperature difference usually appeared after 14:00, which changes with time and orientation.

To some extent, the influence of absorptivity variation is the most sensitive term among solar radiation absorptivity, diameter, wall thickness and geographic latitude. With the increase of the absorptiv- ity and Wall thickness, the temperature difference increases obviously. 
The surface cracks occur due to the large stress which is induced by the extensive sunshine temperature difference. So accurate monitoring on temperature is indispensable, and the data collection is useful for the further research.

\section{References}

[1] Gu Xiaohua translated. Theory and practice of silo. Beijing: china architecture and building press. 1987

[2] Jiang Shufen; Jofriet, J.C.. Finite element prediction of silage temperatures in tower silos. Transactions of the American Society of Agricultural Engineers, 30(1987):1744- 1750.

[3] J.Y.Ooi, J.M.Rotter. Wall pressure in squat steel silos from simple finite element analysis. Computers and structures. (1990).

[4] $\mathrm{Pu}$ Weiming. Temperature effect of reinforced concrete. coal mine design, 8(1999):41-42.

[5] Xia Guangzheng, Xia Dongtao. Finite element analysis of giant reinforced concrete coal silos subject to temperature load. Spatial Structures, 12(2006):62-64.

[6] Fang Hongqiang, Wei Puming. The analysis and calculation on temperature stress in huge reinforced concrete silo [J]. Spatial Structures, 28(2011):53-57.

[7] Yang Yinghua, Zhang Yan. Temperature effects on the buckling of large flat-bottom squat steel silos for alumina. Advances in Steel Structures, Nanjing: Southeast University Press, 2012: 117 3-1178.

[8] Cui Yanrui, Wang Zhouhui. temperature stress of silo structures. Spatial Structures, 29(2012): $1-5$.

[9] Zhaohui Zhang. ANSYS 8.0 thermal analysis tutorial and examples. Beijing: China railway publishing house. 2005. 